\title{
Pulmonary Rehabilitation is Improved by In-Shoe Foot Orthosis Intervention
}

\author{
Authors: \\ *Yves Jammes, ${ }^{1,2}$ Hanan Rkain, ${ }^{3}$ Jean Paul Weber, ${ }^{2}$ Patricia Griffon, ${ }^{2}$ \\ Bruno Vie, ${ }^{2}$ Alain Palot, ${ }^{4}$ Pierre François Gallet ${ }^{5}$ \\ 1. Faculty of Medicine, Aix Marseille University, Marseille, France \\ 2. School of Podiatry, Aix Marseille University, Marseille, France \\ 3. Laboratory of Physiology, Faculty of Medicine, Mohammed V University, \\ Rabat, Morocco \\ 4. Department of Respiratory Medicine, Hôpital Nord, Assistance Publique - Hôpitaux \\ de Marseille, Marseille, France \\ 5. Korian Clinic Les Trois Tours La Destrousse, Bouches du Rhône, France \\ *Correspondence to yves.jammes@univ-amu.fr \\ Disclosure: $\quad$ This work was funded by a grant from Association Nouveau Souffle, France. \\ Acknowledgements: The authors acknowledge Mrs Alexandra Dani and Mrs Neima Ayadi for their helpful \\ assistance in this study. \\ Received: $\quad 08.10 .19$ \\ Accepted: $\quad 15.11 .19$ \\ Keywords: $\quad$ 6-minute walk test (6MWT), foot orthosis, plantar flexion force (PFF), posturography, \\ pulmonary rehabilitation (PR), respiratory insufficiency. \\ Citation: \\ EMJ. 2020;5[1]:41-47.
}

\section{Abstract}

Some studies have found that patients with asthma have bilateral foot dorsal flexion limitations, contributing to impaired quality of life. The authors hypothesised that foot misalignments could also occur in patients with chronic obstructive pulmonary disease (COPD), and that foot orthoses could increase the motor benefits of their pulmonary rehabilitation (PR).

Presented herein are the results from a nonrandomised controlled study in 40 patients with COPD. Twenty patients had foot misalignment (Group 1) and wore foot orthoses for a 1-month PR period. Their data were compared to those obtained in 20 other patients with COPD who had no foot misalignment and did not wear foot orthoses (Group 2). Bodily fatigue, 6-minute walk test (6MWT) distance, peak plantar flexion force (PFF), and oscillations of the centre of pressure (CoP) were measured. Measurements were performed prior to and following completion of PR (Groups 1 and 2), immediately after wearing the foot orthosis (Group 1), and after completion of PR plus foot orthoses (Group 1).

In Group 2, PR increased the 6MWT distance, but did not increase PFF nor reduce CoP oscillations and fatigue scale. Wearing the foot orthosis for the first time significantly increased the 6MWT distance $(+98+12 \mathrm{~m})$. Following PR with foot orthoses (Group 1), a further increase in 6MWT distance occurred $(+120+13 \mathrm{~m})$, bodily fatigue was reduced, PFF increased, and CoP oscillations decreased.

In patients with COPD and foot misalignment, foot orthoses enhanced the functional capacity and improved the postural control. 


\section{INTRODUCTION}

Pulmonary rehabilitation (PR) is currently the most cost-effective approach designed to improve exercise tolerance, peripheral muscle function, and quality of life in patients with chronic obstructive pulmonary disease (COPD)., The clinical management of patients with COPD and peripheral muscle dysfunction, based on improving the functional capacity of skeletal muscles, is widely documented, ${ }^{3,4}$ and the 6 -minute walk test (6MWT) is commonly used to evaluate the benefits of exercise training. ${ }^{5}$

Despite data not being available in a COPD setting, some studies have reported that patients with asthma have increased fall rates as a result of bilateral foot dorsal flexion limitations of the ankle, ${ }^{6}$ contributing to impaired quality of life. ${ }^{7}$ These observations suggest that foot orthoses intervention could improve the ambulatory performance and thus quality of life of patients with respiratory disorders. It has in fact been shown that foot orthoses improve the ambulatory capacities and posture of normal weight and obese patients, ${ }^{8}$ venous return, ${ }^{9}$ and foot sole mechanosensitivity, ${ }^{10}$ an observation also confirmed in patients with asthma. ${ }^{11}$ The inshoe foot orthoses are designed to reduce rear foot misalignment and hold the foot close to its subtalar neutral position in order to restore normal alignment of the entire lower limb.

In the present study, podiatrists diagnosed a major foot misalignment in the authors' COPD population (Group 1). Based on previous findings, ${ }^{8-11}$ it was hypothesised that a foot orthosis intervention supplementing regular PR could induce additional functional benefits to these patients, improving their ambulatory performance and reducing the posture sway. The authors also examined the benefits of regular PR alone in another group of patients with COPD and no foot misalignment (Group 2), who did not wear the foot orthosis.

\section{METHODS}

\section{Study Design}

This was a nonrandomised, controlled study in patients with COPD comparing the consequences of PR alone (Group 1; $n=20$ ) or associated with foot orthoses intervention (Group 2; $n=20$ ) on their capacity to walk and the postural changes. The French institutional review boards for human studies (Agence Nationale de Sécurité du Médicament et des Produits de Santé [ANSM] and Comités de Protection des Personnes [CPP]) approved the study protocol, which followed the principles outlined in the code of ethics of the World Medical Association (WMA) (Declaration of Helsinki). Written informed consent was obtained from all patients. The characteristics of patients are shown in Table 1. All patients were undergoing treatment of their chronic respiratory disease but did not receive oxygen supplementation. Their practitioners had good control of their symptoms. Exclusion criteria was the diagnosis of diabetes, neuromuscular disorders, leg trauma, and spine diseases.

In both groups, PR lasted 4 weeks. All data were measured prior to and after each period of PR. In both groups, the foot configuration was determined by podiatrists based on: 1) the measurement of the distance between the rear foot and forefoot surface plans; 2) the diagnosis of the plantar deformity; and 3) the analyses of the plantar footprint. Group 1 patients had major foot misalignment (navicular drop: 6; high-arch foot: 4; low-arch foot: 5; static disorders due to anisomelia that is a leg length inequality: 5). Assessment prior to PR included pulmonary function tests, cardiopulmonary exercise testing, 6MWT, maximal plantar flexion force (PFF), bodily fatigue scale, and measurements of oscillations of the centre of pressure (CoP). For patients in Group 1, the acute effects of foot orthoses were also assessed prior to PR with foot orthoses.

\section{Pulmonary Function and Gas Exchange}

Forced vital capacity and forced expiratory volume in 1 second were measured spirometrically (MasterLab ${ }^{\odot}$, Levallois-Perret, France). The reference values were those proposed by Quanjer. ${ }^{12}$ Arterial blood gas tensions $\left(\mathrm{PaO}_{2}\right.$ and $\mathrm{PaCO}_{2}$ ) and $\mathrm{pH}(\mathrm{pHa}$ ) were measured by a blood gas analyser (Corning Chiron model 860 ${ }^{\circ}$, Chiron Technologies, Villeuneuve-La-Garenne, France). Cardiopulmonary exercise testing was performed on a cycle ergometer, included in the PR programme to determine the ventilatory threshold and the corresponding heart rate. 


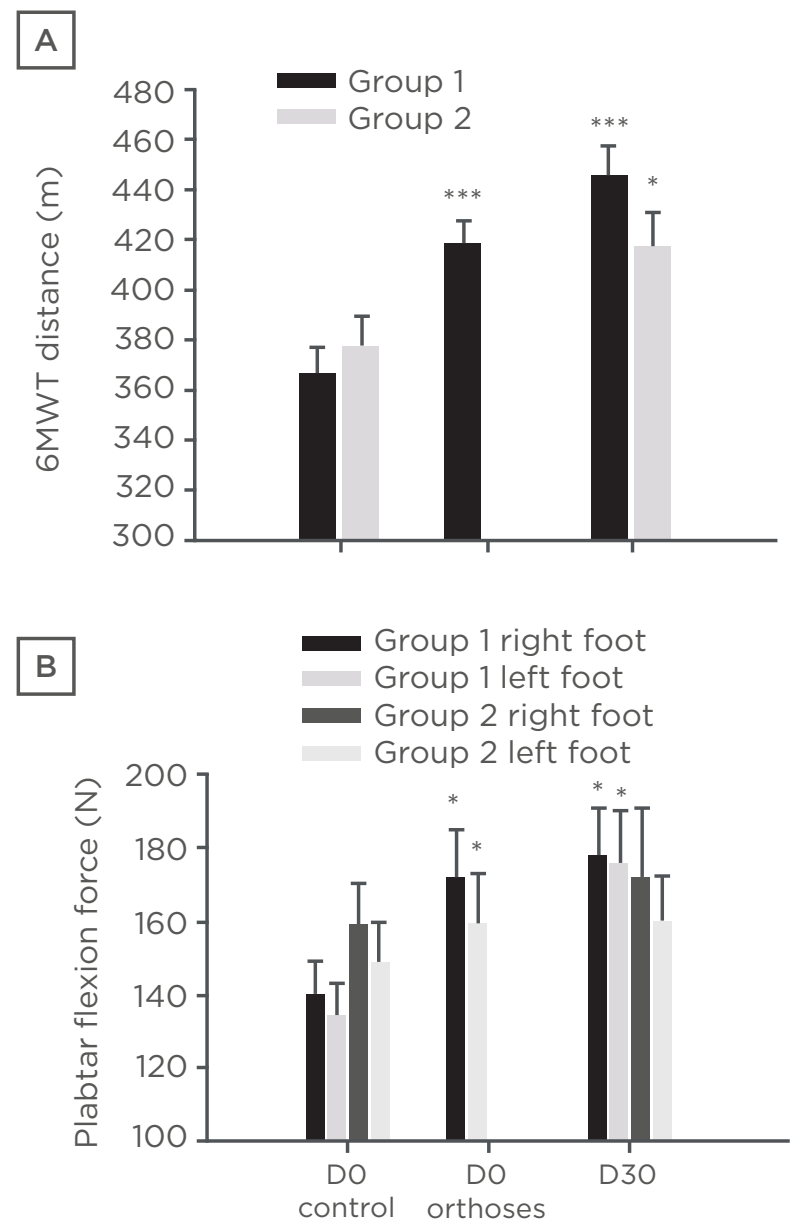

Figure 1: (A) Benefits of foot orthoses intervention on the ambulatory capacities. (B) Peak plantar flexion force. The 6MWT distance significantly increased in Group 1 patients who wore their foot orthoses for the first time to begin pulmonary rehabilitation (DO orthoses) $\left({ }^{* *} \mathrm{p}<0.01\right)$. After completion of pulmonary rehabilitation (D30), this effect increased in Group $1\left({ }^{* * *} \mathrm{p}<0.001\right)$ and Group $2\left({ }^{* *} \mathrm{p}<0.01\right)$. In Group 1 patients, the foot orthoses significantly increased the peak plantar flexion force when they were worn for the first time and also after completion of pulmonary rehabilitation with orthoses $\left({ }^{*} p<0.05\right)$. No benefits of pulmonary rehabilitation on plantar flexion force were noted in Group 2.

D: day; 6MWT: 6-minute walk test.

\section{Pre and Post-PR Measurements}

\section{Maximal Plantar Flexion Force}

Maximal peak PFF was measured under isometric conditions using a custom-built device previously described in detail. 8,13 Three 5-s PFF maneuvers were executed by the subject using each leg to determine the maximal force value. The best values were considered.

\section{Pedobarographic Measurements}

A recent review validated the use of a pedobarographic platform in postural assessment. $^{14}$ Subjects were bare-footed when standing on the platform for 30 seconds (WinPOD Medicapteurs SA ${ }^{\circ}$, Balma, France). Patients were positioned standing on the platform, with lowered arms, heels $2 \mathrm{~cm}$ apart, barefoot, and feet at $30^{\circ}$ so that the centroid of the sustentation polygon was located on the sagittal axis of the platform. Postural oscillations were recorded for 30 seconds. The authors measured postural variables: the peak and mean foot pressures, the surface covered by displacements of the CoP, and its total length of displacement. Measurements of PFF and postural variables were repeated twice. 

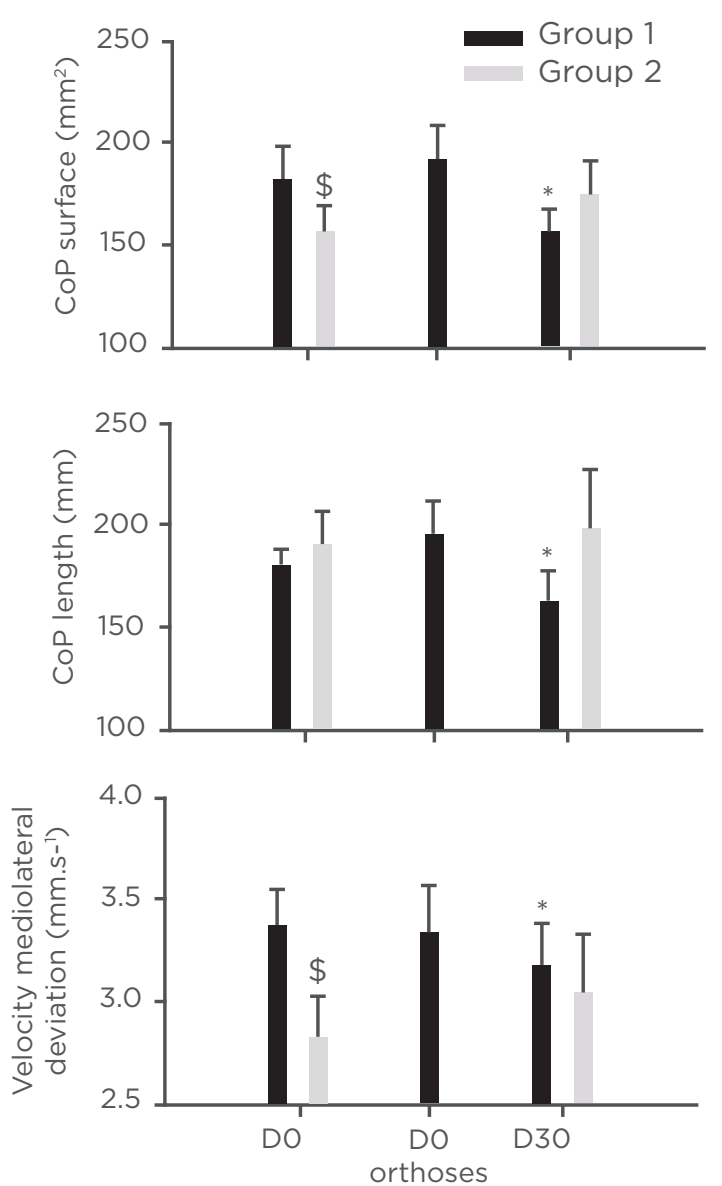

Figure 2: Posturographic indices (surface and length of the CoP and velocity of mediolateral CoP deviation) measured before PR (DO), when wearing foot orthoses before beginning PR (DO orthoses) in Group 1, and after completion of the whole PR programme (D30) in both groups. Wearing foot orthoses during the PR programme significantly reduced the three variables in Group 1. No effects were measured in Group 2 patients.

CoP: centre of pressure; D: day; PR: pulmonary rehabilitation.

\section{6-Minute Walk Test}

As recommended by the American Thoracic Society (ATS), ${ }^{5} 1$ hour after the first 6MWT, a second test was performed during which all the measurements were considered.

\section{Sensations of Bodily Fatigue}

The patients addressed the questions of the Pichot bodily fatigue scale (validated for French subjects). ${ }^{15}$

\section{Pulmonary Rehabilitation Programme}

All patients participated in a comprehensive 4-week PR programme based on the updated definition proposed by the ATS-European Respiratory Society (ERS) group consensus. ${ }^{2}$ The training programme included endurance exercise of both interval and continuous modalities (cycling and walking), at a training intensity corresponding to a heart rate of $80 \%$ of that measured at the ventilatory threshold during a maximal cardiopulmonary exercise test. PR also included peripheral muscle strength training of upper and lower limbs, and upper extremity dynamic exercises on an arm cycle ergometer three to five times per week for 60 minutes each time.

\section{Custom-Molded Foot Orthoses}

Thermoformed pads were built and middle density (20-30 Shore) materials were placed on selected sole locations to correct the foot misalignment. Depending on the type of foot abnormality, retro capital bars and/or foot arch reinforcement were added. 


\section{Statistical Analyses}

Based on mean and standard error of the mean data from previous studies that explored the consequence of foot orthoses intervention, ${ }^{8}$ and the 6MWT data ${ }^{13}$ on the postural control and PFF values in normal and excess weight subjects, the authors estimated that 18 patients were necessary for each group. A two-way repeated measures ANOVA test allowed depiction of significant changes of the variables across the different time points between the two PR conditions (PR alone versus PR plus foot orthoses). When the normality test failed, the pairwise multiple comparison procedure (Holm-Sidak method) was used. With the numbers available, no significant difference could be detected when $p>0.05$.

\section{RESULTS}

\section{Intergroup Differences at Baseline}

At study entry, pulmonary function (forced expiratory volume in 1 second and $\mathrm{PaO}_{2}$ values) and exercise tolerance (maximal $\mathrm{O}_{2}$ uptake) did not differ between groups (Table 1). On the contrary, compared to Group 2, the bodily fatigue scale was significantly higher in Group 1 patients (13.7 \pm 2.2 versus $8.0 \pm 3.0 ; p<0.001)$, and their 6MWT distance (Figure 1A) and PFF (Figure 1B) were lower. Moreover, the postural control of Group 1 patients was less efficient than that of Group 2 (Figure 2), with higher CoP surface and velocity of mediolateral CoP deviation.

Table 1: Patient characteristics.

\begin{tabular}{|c|c|c|c|c|}
\hline & Group 1 & & Group 2 & \\
\hline & PRE & POST & PRE & POST \\
\hline $\mathrm{n}$ & 20 & & 20 & \\
\hline Sex ratio & $7 / 20$ & & $5 / 20$ & \\
\hline Age, years & $64 \pm 2$ & & $67 \pm 2$ & \\
\hline Weight (kg) & $77 \pm 4$ & & $68 \pm 5$ & \\
\hline Height $(\mathrm{cm})$ & $169 \pm 1$ & & $166 \pm 2$ & \\
\hline BMI & $27.0 \pm 1.4$ & & $24.6 \pm 1.6$ & \\
\hline \multirow[t]{2}{*}{ FEV ${ }_{1}, \mathrm{BTPS}$} & $1.43 \pm 0.14$ & $1.57 \pm 0.14$ & $1.27 \pm 0.11$ & $1.34 \pm 0.12$ \\
\hline & $(53 \pm 4)$ & $(57 \pm 4)$ & $(49 \pm 4)$ & $(53 \pm 5)$ \\
\hline \multirow[t]{2}{*}{ FVC, BTPS } & $2.66 \pm 0.15$ & $2.97 \pm 0.16$ & $2.40 \pm 0.15$ & $2.51 \pm 0.19$ \\
\hline & $(77 \pm 4)$ & $(80 \pm 4)$ & $(70 \pm 4)$ & $(74 \pm 6)$ \\
\hline FEV1.0/VC (\%) & $54 \pm 3$ & $56 \pm 3$ & $56 \pm 2$ & $56 \pm 3$ \\
\hline $\mathrm{PaO}_{2}(\mathrm{mmHg})$ & $77 \pm 2$ & $79 \pm 2$ & $78 \pm 2$ & $75 \pm 2$ \\
\hline $\mathrm{PaCO}_{2}(\mathrm{mmHg})$ & $40 \pm 1$ & $39 \pm 1$ & $36 \pm 1$ & $37 \pm 1$ \\
\hline $\mathrm{pHa}$ & $7.40 \pm 0.01$ & $7.42 \pm 0.001$ & $7.42 \pm 0.01$ & $7.43 \pm 0.01$ \\
\hline $\begin{array}{l}\mathrm{VO}_{2} / \text { body weight } \\
\left(\mathrm{ml} \mathrm{min}^{-1} \cdot \mathrm{kg}^{-1}\right)\end{array}$ & $19.5 \pm 1.1$ & & $18.5 \pm 1.4$ & \\
\hline $\begin{array}{l}\text { HR threshold beats } \\
\left(\mathrm{min}^{-1}\right)\end{array}$ & $116 \pm 4$ & & $115 \pm 5$ & \\
\hline
\end{tabular}

Variables measured at inclusion in the study (PRE) and after completion of the 8-week rehabilitation program (POST).

Peak oxygen uptake ( $\mathrm{VO}_{2} /$ body weight) and heart rate measured at the ventilatory threshold. Values are the mean \pm standard error of mean. Values in parentheses are the percentage of predicted FEV1 and FVC.

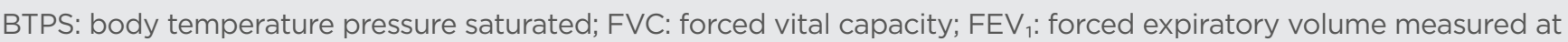
1.0 s; HR: heart rate; $\mathrm{PaO}_{2}$ and $\mathrm{PaCO}_{2}$ : partial pressures of oxygen and carbon dioxide in arterial blood; VC:

vital capacity. 


\section{Foot Orthoses Intervention and Bodily Fatigue Sensation}

At the end of the PR programme, compared to data at Day 0 , a significant reduction of bodily fatigue scale was measured in Group 1 (9.2 \pm 2.4 versus $13.7 \pm 2.2$ ), whereas there was only a trend to a reduction in Group 2 (7.2 \pm 2.8 versus 8.0 \pm 3.0$)$.

\section{Foot Orthoses Intervention and the 6-Minute Walk Test Distance}

In Group 1, wearing the foot orthosis for the first time prior to beginning PR significantly increased the 6MWT distance $(+55 \pm 12 \mathrm{~m})$ (Figure 1A). Following PR completion with foot orthoses, a further increase in 6MWT distance was noted $(+95 \pm 13 \mathrm{~m})$, however the changes were not significant. PR alone also significantly increased 6MWT distance in Group 2 but to a lesser extent $(+40 \pm 8 \mathrm{~m})$.

\section{Foot Orthoses Intervention and Plantar Flexion Force}

The benefits of the foot orthosis on PFF values occurred soon after the Group 1 patients wore their foot orthoses for the first time. At the end of PR with foot orthoses, PFF of both feet continued to be increased but no further changes were measured. No significant PPP variations occurred in Group 2 (Figure 1B).

\section{Foot Orthoses Intervention and Postural Control}

No significant postural changes were noted in Group 1 patients after they wore their foot orthoses for the first time. On the other hand, after completion of the 4-week PR programme, the CoP surface and length, and also the velocity of mediolateral CoP deviation, were significantly reduced. No significant variation of the CoP characteristics was noted after PR completion in Group 2 (Figure 2).

\section{DISCUSSION}

The authors found ergonomic differences between the two groups at inclusion in the study. In patients who presented foot misalignments at study entry, the fatigue scale was significantly higher, the PFF values significantly lower, and their postural control less efficient, with higher CoP surface and higher velocity of mediolateral CoP deviation. Thus, foot misalignment reduced the ambulatory performance of Group 1 patients with COPD. These data confirm the observations of reduced ambulatory capacities in patients with asthma. ${ }^{6,7}$

The present study shows in Group 1 patients with COPD experiencing foot abnormalities that foot orthoses reduced bodily fatigue, markedly increased the 6MWT distance and the PFF, and improved postural control. PR alone in the Group 2 patients increased the 6MWT distance to a lesser extent but did not increase PFF nor reduce the CoP oscillations and the fatigue scale.

Several studies in healthy subjects and in patients with neuromuscular disorders support the hypothesis that foot orthoses intervention may help walking. In healthy subjects, foot orthoses improve the biomechanical capacities of the ankle, $8,16,17$ increase the tactile sensitivity of the foot sole, ${ }^{10}$ and improve the postural control., 8,18 Based on these observations, wearing foot orthoses has been proposed as a support of the functional rehabilitation of patients with chronic muscle dystrophy, ${ }^{19}$ spinal muscular atrophy, ${ }^{20}$ or chronic stroke. ${ }^{21}$

Most activities of daily living are performed at submaximal levels of exertion and this is reproduced during a PR programme. Moreover, the 6MWT reflects the functional capacity for daily physical activities. ${ }^{5}$ Several possible causes for the improvement of ambulatory capacities by foot orthoses have been previously identified. Firstly, foot orthoses were suggested to increase the strength of the foot muscles participating in plantar flexion, the benefits of which are still presenting in this study. This effect was already reported, ${ }^{22}$ showing that medially posted insoles consistently influenced the foot pronation. The authors recently confirmed these observations showing that the addition of $3 \mathrm{~mm}$ heel pads significantly increased the peak PFF, probably through an increased lever arm exerted by the rearfoot on the forefoot. ${ }^{8}$ Secondly, the present study in patients with COPD confirmed the previous observation in healthy subjects that foot orthoses improved posture control; this effect could have contributed to the increased 6MWT distance. ${ }^{8}$ This improved postural control may 
also result from an increased mechanosensitivity of the foot sole, induced by the foot orthoses. ${ }^{10}$ The cutaneous mechanoreceptors of the foot sole detect changes in the application of mechanical loads on the plantar surface during gait and standing, and contribute to controlling the standing balance and postural reflexes in healthy subjects. ${ }^{18}$

\section{CONCLUSION}

One message of this study is that foot orthoses markedly improve ambulatory capacities. Patients with COPD wearing foot orthoses benefitted by ergonomic improvements during $\mathrm{PR}$, including increased leg muscle force and improved postural control. In a future study it would be interesting to compare a case-control group on the use of plantar support. Regardless, the present data strongly recommend that clinicians should routinely examine their patients that have COPD for foot abnormalities. However, the benefits of foot orthoses on rehabilitation is almost certainly not limited to a COPD population. It is likely that in any population with significant foot misalignment, treatment targeting the foot disorder would improve the walking distance and postural control.

\section{References}

1. Maltais F et al. An official American Thoracic Society/European Respiratory Society statement: update on limb muscle dysfunction in chronic obstructive pulmonary disease. Am J Respir Crit Care Med. 2014;189:15-62.

2. Rochester $\mathrm{CL}$ et al. An official American thoracic society/European Respiratory Society policy statement: enhancing implementation, use, and delivery of pulmonary rehabilitation. Am J Respir Crit Care Med. 2015;192:1373-86.

3. Gea J et al. Clinical management of chronic obstructive pulmonary disease patients with muscle dysfunction. J Thorac Dis. 2016;8(11):3379-400.

4. Gea J et al. Muscle dysfunction in chronic obstructive pulmonary disease: update on causes and biological findings. J Throrac Dis. 2015;7(10):E418-38.

5. Singh SJ et al. An official systematic review of the European Respiratory Society/American Thoracic Society: measurement properties of field walking tests in chronic respiratory disease. Eur Respir J. 2014;44(6):1447-78

6. Calvo-Lobo $\mathrm{C}$ et al. Falls rate increase and foot dorsal flexion limitations are exhibited in patients who suffer from asthma: a novel case-control study. Int J Med Sci. 2019;16(4):607-13.

7. López-López D et al. Impact of the allergic asthma on foot health-related quality of life and depression: a novel case-control research. Medicina (Kaunas). 2019;55(5):124.

8. Jammes $Y$ et al. Four weeks of foot orthosis intervention improves ambulatory capacities and posture of normal weight and obese patients. J Am Podiatr Med Assoc. 2020;110)1):Article 2.

9. López-López D et al. Influence of custom foot orthoses on venous status: a quasi-experimental study. $\mathrm{J}$ Mech Behav Biomed Mater. 2018;79:235-8

10. Vie B et al. Pilot study demonstrating that sole mechanosensitivity can be affected by insole use. Gait Posture. 2015;41:263-8.

11. Calvo-Lobo $\mathrm{C}$ et al. Tarsal tunnel mechanosensitivity is increased in patients with asthma: a case-control study. J Clin Med. 2018;7(12):541.

12. Quanjer PH. Standardized lung function testing. Bulletin Europeen de Physiopathologie Respiratoire. 1983;19(Suppl 5):7-44.

13. Vie B et al. Effect of the 6-minute walk test on plantar loading and capability to produce ankle plantar flexion forces. Gait Posture. 2016;49:61-6.

14. Rosário JL. A review of the utilization of baropodometry in postural assessment. J Bodyw Mov Ther. 2014;18(2):215-9.
15. Pichot $V$ et al. Quantification of cumulated physical fatigue at the workplace. Pflügers Arch. 2002;445(2):267-72

16. Lehmann JF. Push-off and propulsion of the body in normal and abnormal gait. Correction by ankle-foot orthoses. Clin Orthop Relat Res. 1993;288:97-108

17. Shelburne KB et al. Effects of foot orthoses and valgus bracing on the knee adduction moment and medial joint load during gait. Clin Biomech (Bristol, Avon). 2008;23(6):814-21.

18. Wu G, Chiang JH. The significance of somatosensory stimulations to the human foot in the control of postural reflexes. Exp Brain Res. 1997;114(1):163-9.

19. Siegel IM. The management of muscular dystrophy: A clinical review. Muscle Nerve. 1978;1(6):453-60.

20. Montes J et al. Six-minute walk test demonstrates motor fatigue in spinal muscular atrophy. Neurology. 2010;74(10):833-8.

21. Bouchalová $V$ et al. The influence of an ankle-foot orthosis on the spatiotemporal gait parameters and functional balance in chronic stroke patients. J Phys Ther Sci. 2016;28(5):1621-8.

22. Rodrigues $P$ et al. Medially posted insoles consistently influence foot pronation in runners with and without anterior knee pain. Gait Posture. 2013;37(4):526-31. 